\title{
The stellar populations of host galaxies of supernovae
}

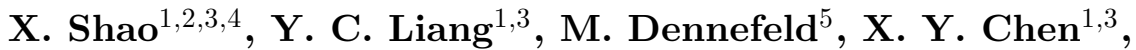 \\ G. H. Zhong ${ }^{1,3}$, F. Hammer ${ }^{6}$, L. C. Deng ${ }^{1,3}$, and B. Zhang ${ }^{1,4}$ \\ ${ }^{1}$ National Astronomical Observatories, CAS, 20A Datun Road, 100012, Beijing, PR China \\ email: xshao@bao.ac.cn \\ ${ }^{2}$ University of Chinese Academy of Sciences, 19A Yuquan Road, 100049, Beijing, PR China \\ ${ }^{3}$ Key Laboratory of Optical Astronomy, NAOC, 20A Datun Rd. 100012, Beijing, China \\ ${ }^{4}$ Department of Physicals, Hebei Normal University, Shijianzhuang 050016, China \\ ${ }^{5}$ Institut d'Astrophysique de Paris, CNRS, 98bis Bd Arago, F-75014 Paris, France \\ ${ }^{6}$ GEPI,Observatoire de Paris-Meudon, 92195 Meudon, France
}

\begin{abstract}
We study and compare the stellar populations of host galaxies of different types of supernovae (SNe): SN Ia and core collapse SN (SN II and SN Ibc) at the same time. The 234 sample galaxies are selected by cross-matching the Asiago Supernova Catalogue (ASC) and the SDSS-DR7 main galaxy sample (MGS). The STARLIGHT software is used to analyze their stellar populations by fitting the continua and absorption lines of the hosts.
\end{abstract}

Keywords. galaxies: evolution, galaxies: star formation, galaxies: starburst

We performed cross-matching on the ASC and the SDSS-DR7 MGS with 30 arcsec radius to select supernova host galaxies. We select galaxies for which the light-fraction (see details in Liang et al. 2010) of their SDSS spectral observations are $>0.15$ to ensure that the 3 arcsec fiber can cover most of their global light. In total $234 \mathrm{SN}$ host galaxies are selected, which are divided into two subsamples: emission-line galaxies and absorptionline galaxies. We fit the stellar continua and absorption lines of the hosts using Starlight (Cid Fernandes et al. 2005, Chen et al. 2009). The results are shown in Table 1. Among the 137 emission-line galaxies, the fraction of young stellar populations is higher in hosts of SN II than in hosts of SN Ia and Ibc. Mots of the 97 absorption-line galaxies host a SN Ia, and they have a large fraction of old stellar populations. The 137 hosts with emission lines contain much younger stellar populations.

Table 1. The contributed light fraction of stellar populations in age-bins for SN host galaxies.

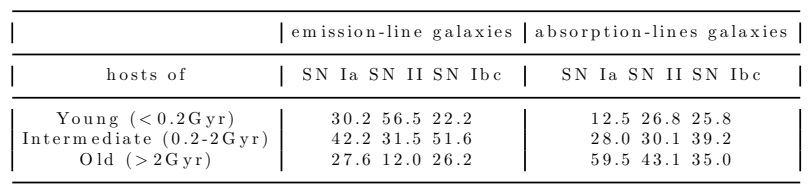

Acknowledgements. The authors thank the symposium organizers for their invitation to this poster. This work was supported by the Natural Science Foundation of China (NSFC) Foundation under Nos.10933001, 11273026.

\section{References}

Chen, X. Y., Liang, Y. C., Hammer, F. et al. 2009, A\& $A$, 495, 457

Cid Fernandes, R., Mateus, A., Sodre, L. et al. 2005, MNRAS, 358, 363

Liang, Y. C., Zhong, G. H., Hammer, F. et al. 2010, MNRAS, 409, 213 